\title{
Influence of Running Shoes and Running Velocity on "Ride" during Running ${ }^{\dagger}$
}

\author{
Franziska Mally *, Otto Hofstätter and Markus Eckelt \\ Department of Life Science Engineering, University of Applied Sciences Technikum Wien, 1200 Vienna, \\ Austria; hofstaet@technikum-wien.at (O.H.); eckelt@technikum-wien.at (M.E.) \\ * Correspondence: mally@technikum-wien.at; Tel.: +43-1-333-40-77-8770 \\ + Presented at the 13th conference of the International Sports Engineering Association, Online, 22-26 June 2020.
}

Published: 15 June 2020

\begin{abstract}
Ride" has been established to subjectively describe the heel-to-toe transition during walking and running. Recently, a study was published aiming to quantify "ride" by linking it to the maximum velocity of the anterior-posterior $(\mathrm{AP})$ progression of the center of pressure (COP) during the first $30 \%$ of the stance phase. While that study investigated the parameter when running at a constant velocity of approximately $3.5 \mathrm{~m} / \mathrm{s}$ (i.e., $12.6 \mathrm{~km} / \mathrm{h}$ ), this study was carried out to evaluate the influence of running velocity on "ride" when running. Five healthy participants performed runs on a treadmill at 8,10 and $12 \mathrm{~km} / \mathrm{h}$ with three different running shoes, and their plantar pressure was measured at $300 \mathrm{~Hz}$ using pressure-sensing insoles. "Ride" was calculated as suggested by the previously mentioned study. In two of the three shoes, "ride" decreased with increasing running speed. Between the shoes, however, there is no clear image of how the shoes influence this parameter.
\end{abstract}

Keywords: running; velocity; footwear; plantar pressure; "ride"

\section{Introduction}

Comfort in general and, more specifically, footwear comfort has been reported to be highly subjective and hard to impossible to measure [1-7]. While a certain group of people can consider one type of shoe comfortable, others can consider the same shoe uncomfortable. Nevertheless, comfort has been suggested to have a possibly positive influence on injury reduction during physical activities [5]. Furthermore, Luo et al. [7] found that subjectively more comfortable shoes improve running economy by an average of $0.7 \%$ decrease in oxygen consumption.

While comfort itself can be assessed using different measures, such as a Borg scale [4], visual analogue scales [5,6] or simple rankings [3,7], its clear link to biomechanical parameters is limited. For the walking movement, certain parameters of plantar pressure measurements could be found, related to the subjective comfort of a running shoe. For running, however, these findings could not be confirmed [3].

Quite recently, Lam and colleagues [8] have proposed a method to quantify the so-called "ride", which the authors defined as being related to a smooth feeling during the transition from heel to toe during heel-toe running. Hence, they expected a possible relationship between "ride" and the velocity trace of the center of pressure (COP) movement in the anterior-posterior (AP) direction (COP_AP). For the quantification of "ride", the path of the COP_AP is differentiated, which "portrays the speed at which the CoP is moving across the foot" [8] (p. 78).

It has further been shown that this parameter peaks within the first $30 \%$ of the stance phase (StP) [9]. Interestingly, in all participating subjects, Lam et al. [8] found out that a shoe which was subjectively rated higher in comfort showed a lower peak velocity of the COP_AP within the first $30 \%$ of the StP during running. All subjects but one rated the shoe with the lower velocity of the 
COP_AP higher in terms of "ride". Hence, "ride" could be described by a lower velocity of the COP_AP within the first $30 \%$ of the StP.

Since the study was carried out only at a constant running speed of $3.5 \mathrm{~m} / \mathrm{s}$ (i.e., $12.6 \mathrm{~km} / \mathrm{h}$ ) and running speed has previously been reported to influence several parameters of plantar pressure measurements [10,11], the present study aimed to investigate the influence of running speed on the recently presented calculation of "ride". Furthermore, as in [8], different shoe types were investigated, whereas comfort was not explicitly rated by the participants at that stage of study design.

\section{Materials and Methods}

All measurements for this study took place in the Sports Technology laboratory at the University of Applied Sciences Technikum Wien (Vienna, Austria).

\subsection{Participants}

A total of five physically active participants ( 4 male/ 1 female; age: $35.8 \pm 6.9$ years; height: $178.6 \pm$ $8.0 \mathrm{~cm}$; weight: $76.2 \pm 8.6 \mathrm{~kg}$; see Table 1) voluntarily took part in this study based on given oral consent, and no further ethical approval was requested. None of them had any current injuries of the lower extremities at the time of measurements. However, one subject suffered a rupture of the anterior crucial ligament two years earlier and another subject suffered a rupture of the Achilles tendon three years prior to the measurements. Possibly relevant injuries of all other participants date back to more than seven years prior to the measurements. At the time of data collection, only one participant mentioned running as the main form of physical activity, and all participants, when running, practiced heel-to-toe running.

Table 1. Participant characteristics.

\begin{tabular}{cccccc}
\hline & Sex & Age [years] & Height [cm] & Weight [kg] & Shoe Size [EU] \\
\hline P1 & m & 36 & 177 & 78 & 42 \\
P2 & $\mathrm{f}$ & 30 & 170 & 74 & 41 \\
P3 & m & 36 & 190 & 89 & 43 \\
P4 & m & 30 & 183 & 75 & 43 \\
P5 & m & 47 & 173 & 65 & 43 \\
\hline
\end{tabular}

\subsection{Experimental Protocol}

The participants had to perform three runs on a treadmill $(\mathrm{h} / \mathrm{p} / \operatorname{cosmos}$ quasar $4.0, \mathrm{~h} / \mathrm{p} / \operatorname{cosmos}$ sports \& medical GmBH, Nussdorf-Traunstein, Germany), and each run was performed with a different running shoe at three different running velocities: 8,10 and $12 \mathrm{~km} / \mathrm{h}$. The running shoes that were tested in this study were the Asics GEL-NIMBUS 18 (ASICS Corporation, Kobe, Japan), the Brooks GLYCERIN 14 (Brooks Sports Inc., Seattle, WA, USA) and the On Cloudflyer (On AG, Zurich, Switzerland). While the midsole geometry of the Asics and Brooks running shoes can be considered conventional, the geometry of the On running shoe has an open-cell technology that is built-up with twelve so-called "clouds" (Figure 1).

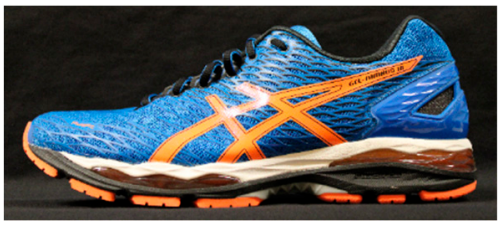

(a)

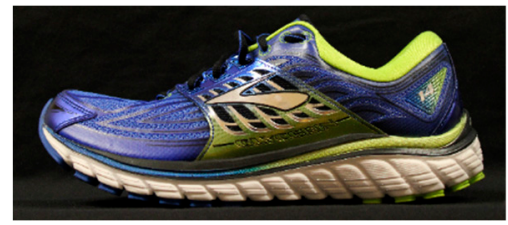

(b)

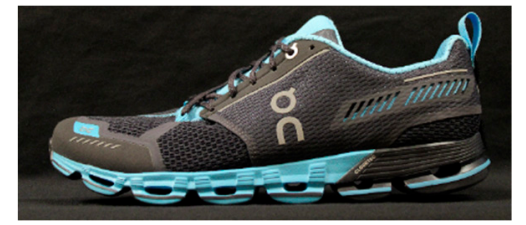

(c)

Figure 1. (a) Asics GEL-NIMBUS 18, (b) Brooks GLYCERIN 14, (c) On Cloudflyer.

The order in which the shoes were chosen was randomized, whereas the order of running velocity was kept constant for every run. The participants were instructed to jump onto the already 
moving treadmill belt at the lowest velocity, and the runs were interrupted for a short moment in order to increase the speed of the belt. Each velocity was kept constant for about $45 \mathrm{~s}$.

The study design did not follow a blinded study, hence the participants could see the shoe model they were wearing at the time. However, they were not specifically instructed about the detailed mechanical differences between the shoes.

Before each run, medilogic pressure-sensing insoles (T\&T medilogic Medizintechnik GmbH, Schönefeld, Germany) were placed in both shoes, on top of the shoes' standard insoles. While the participants were running, plantar pressure data were collected from both feet at a recording frequency of $300 \mathrm{~Hz}$, whereas only data of the right foot were considered for further data treatment. Soles and cables were fixed to the participants' legs using elastic Velcro straps, so they would not obstruct them while running, and the participants had to wear a portable patient modem around their waist, from which the data were wirelessly transmitted to a PC. Raw data were stored as *.csv files, including pressure data of every sensor of both insoles and the gait line (i.e., COP) of both feet.

\subsection{Data Analysis}

Data treatment was done in MATLAB R2019a (The Mathworks Inc., Nattick, MA, USA) and prepared separately for each participant.

At first, data were imported, and the coordinates of the COP were converted from DirX to mm based on the sensor size and inter-sensor distance according to the manufacturer. The start and end of 15 consecutive StPs per run and per velocity were detected from the COP_AP data, and the first five cycles of each run and velocity were neglected. "Ride" was then calculated according to [8]. Hence, every cycle was time-normalized to $100 \%$ of the StP, and the maximum of the COP_AP velocity trace within the first $30 \%$ of the StP was evaluated. For each participant, the mean of 15 cycles was calculated for each run and each velocity.

The resulting values of all five participants were then put into grouped boxplots.

\section{Results}

In the following figures, each box is created from the mean peak velocity of the COP_AP during the first $30 \%$ of the StP of all five participants. The red line indicates the median value, whereas the box itself indicates the 25th to 75th percentile. Whiskers indicate the most extreme data points, in which outliers are not included.

Generally, the present results range between approximately 3 to $6.8 \mathrm{~mm} / \% \mathrm{StP}$, which is in accord to the findings of [8].

The boxplots in Figure 2 show the influence of the three running velocities on the peak velocity of the COP_AP during the first $30 \%$ of the StP for all three tested running shoes. Each running shoe shows a group of three boxplots, and in each group the running velocity increases from left (bright) to right (dark).

When considering the median peak velocity of the COP_AP, the results for the Asics and the Brooks running shoe propose a negative influence of running velocity on "ride" (i.e., lower peak velocity of the COP_AP). The faster the running velocity, the higher the median value for those two shoes. Running with the On Cloudflyer led to the exact opposite results. There, the "ride" appeared to increase with the increase of the running speed. Furthermore, the overall results for the Asics shoe seem to be the most consistent within the five participants.

Looking only at the most extreme data points, the On Cloudflyer showed the highest results at 8 and $12 \mathrm{~km} / \mathrm{h}$.

In Figure 3, comparable to the previous results, the boxplots show the influence of the three running shoes on the peak velocity of the COP_AP during the first $30 \%$ of the StP for all three tested running velocities. Each running velocity shows a group of three boxplots, and each group contains the results of all three shoes. 


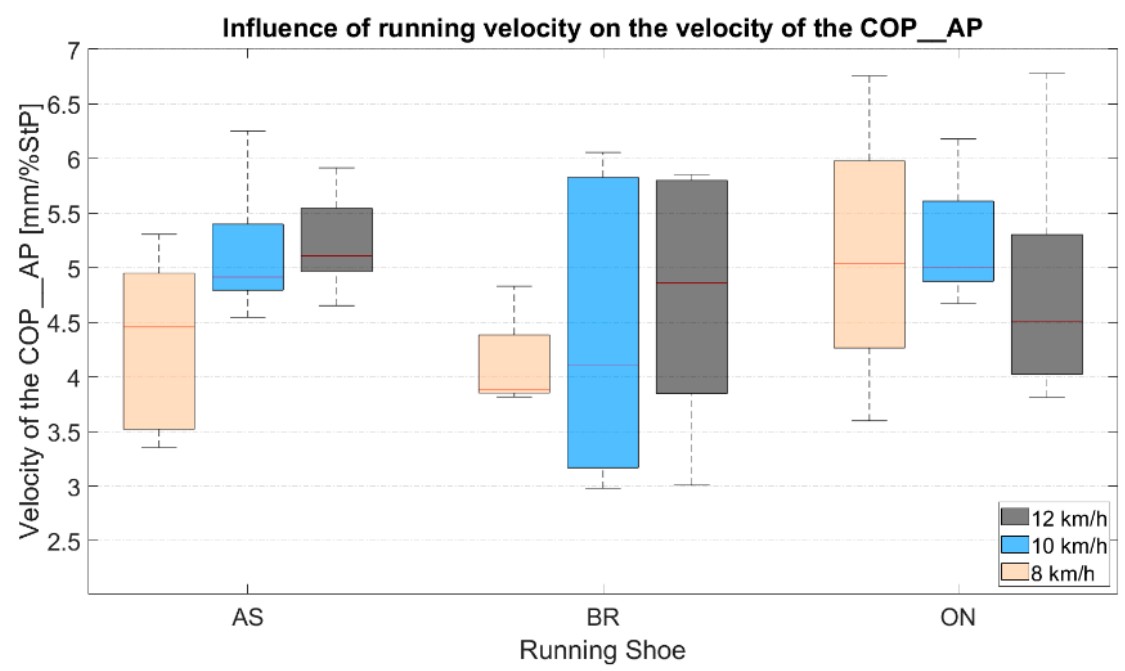

Figure 2. Results for the influence of the three running velocities on the peak velocity of the center of pressure movement in the anterior-posterior direction (COP_AP) during the first $30 \%$ of the stance phase (StP) for all three tested running shoes. The horizontal axis lists the three running shoes (ASAsics, BR-Brooks, ON-On), and the vertical axis shows the "ride" in mm per \% of the StP. The bright flesh-colored boxes represent the lowest running velocity $(8 \mathrm{~km} / \mathrm{h})$, the blue boxes represent the medium running velocity $(10 \mathrm{~km} / \mathrm{h})$, and the dark grey boxes represent the highest running velocity $(12 \mathrm{~km} / \mathrm{h})$.

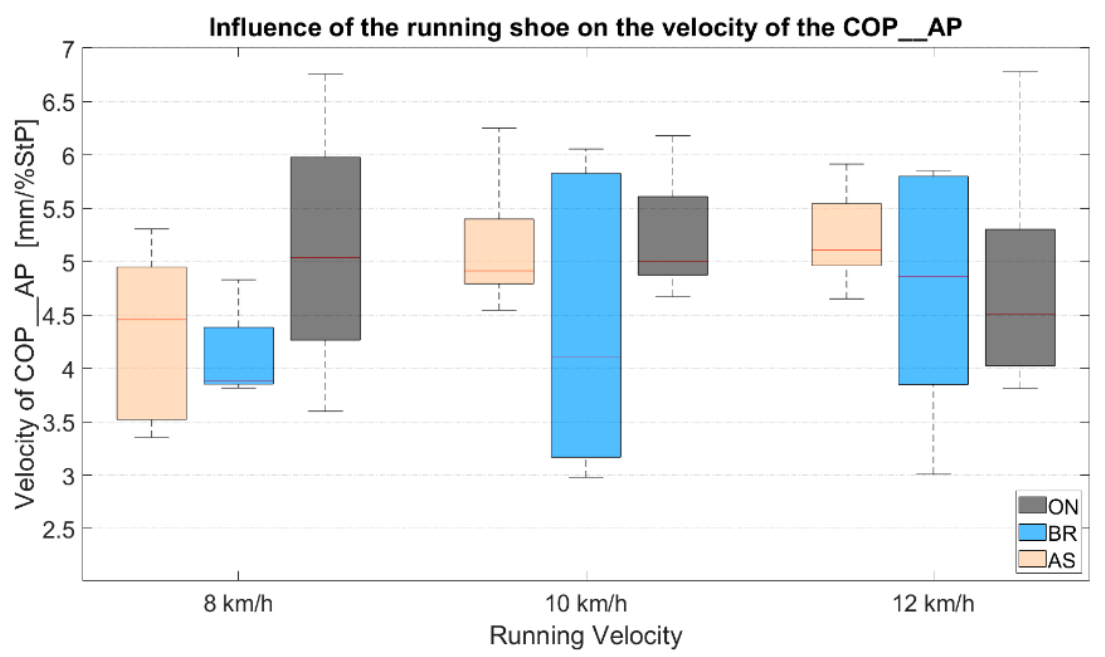

Figure 3. Results for the influence of the three running shoes on "ride" for all three tested running velocities. The horizontal axis lists the three running velocities $(8,10$ and $12 \mathrm{~km} / \mathrm{h})$, and the vertical axis shows the "ride" in mm per \% of the StP. The bright flesh-colored boxes represent the Asics GELNIMBUS 18, the blue boxes represent the Brooks Glycerin 14, the and dark grey boxes represent the On Cloudflyer.

When running at low velocities, the On Cloudflyer showed a higher peak velocity of the COP_AP and, therefore, a lower "ride" compared with the two other shoe models, but it is closely followed by the Asics shoe. The Brooks shoe showed the lowest results. At the highest running speed, however, the On Cloudflyer led to the lowest result in terms of the median value. Again, when considering only the most extreme data points, the results look quite different.

\section{Discussion}

First of all, the quantification of "ride" as presented by Lam et al. [8] was quite comprehensible and reproducible. In general, the results of this study indicate that there is a certain influence of running velocity on the "ride" parameter, which was here evaluated. However, the findings seem to be 
dependent on the shoe type in terms of mid-sole construction. Probably, contrary to what one would expect, when running with conventional running shoes "ride" tends to decrease with increasing running speed, whereas the increase in the peak velocity of the COP_AP could easily be explained by a generally lower StP duration in faster running, and therefore a quicker transition from heel to toe. The findings for the On running shoe lead to the assumption that an interrupted midsole geometry as that of the Cloudflyer (and other current models of On running shoes) leads to a certain inconsistency of the COP_AP transition and therefore to different results.

In a preceding study, the mechanical properties of the three tested shoe models were evaluated via a quasi-static compression test under two loading conditions (400 and $1650 \mathrm{~N}$ ) and an impact test according to the ASTM F1976. Looking at the mechanical properties of the heel portion of the sole (Table 2), it was revealed that, for the compression tests, the Asics shoe was always the softest, followed by the Brooks and the On Cloudflyer. A similar impression was given by the results of the impact test, where the On Cloudflyer was clearly shown to have a hardest sole, whereas the Asics and Brooks shoes showed similar values.

Table 2. Results of mechanical tests of the three running shoes under three test conditions. The values represent the mean displacement in $\mathrm{mm}$, evaluated after ten cycles per test condition.

\begin{tabular}{cccc}
\hline \multicolumn{4}{c}{ Displacement [mm] } \\
\hline & Compression 400 N & Compression 1650 N & Impact \\
\hline Asics & $4.63 \pm 0.33$ & $16.61 \pm 0.17$ & $11.27 \pm 0.53$ \\
Brooks & $4.27 \pm 0.08$ & $16.35 \pm 0.84$ & $11.27 \pm 0.86$ \\
On Cloudflyer & $3.34 \pm 0.38$ & $13.09 \pm 0.53$ & $8.63 \pm 0.51$ \\
\hline
\end{tabular}

Especially when considering the results in Figure 2, it is interesting that the two shoes with the softer sole lead to a decrease of "ride" with increasing running velocity. However, even though the results of the mechanical tests between the Asics and the Brooks are rather similar and the "ride" shows a similar pattern of dependence on running velocity for those two shoes, the Brooks shoe shows the highest overall "ride" for slower running velocities (Figure 3).

\section{Conclusions}

When running with conventionally built running shoes, the results of this study indicate that the "ride" decreases with increasing running speed. Running shoes with an open-cell geometry, such as the On Cloudflyer, seem to have a positive influence on "ride", especially at higher running velocities.

Combining that with the findings of Lam et al. [8] and Mündermann, Stefanyshyn and Nigg [5], one could argue that running with a shoe like the On Cloudflyer would lead to more comfort at higher running velocities, and therefore to a lower risk of injuries. However, due to the very small population of participants in this study and no comparable findings with this type of running shoes, the results can only be seen as a first indication for future research. It would be interesting to repeat the testing with even more different models of both conventional running shoes of different manufacturers and On models, which are all built with a similar open-cell structure. Furthermore, within a follow-up study, comfort should be assessed, since from the current results it seems very questionable whether the conclusion drawn in [8] applies to this special midsole structure.

Conflicts of Interest: The authors declare no conflicts of interest. This study was conducted without external sources of funding.

\section{References}

1. Cavanagh, P.R. The Running Shoe Book; Anderson World Inc.: Mountain View, CA, USA, 1980.

2. Slater, K. Human Comfort; C. C. Thomas: Springfield, IL, USA, 1985.

3. Chen, H.; Nigg, B.M.; De Koning, J. Relationship between plantar pressure distribution under the foot and insole comfort. Clin. Biomech. 1994, 9, 335-341. 
4. Miller, J.E.; Nigg, B.M.; Liu, W.; Stefanyshyn, D.J.; Nurse, M.A. Influence of foot, leg and shoe characteristics on subjective comfort. Foot Ankle Int. 2000, 21, 759-767.

5. Mündermann, A.; Stefanyshyn, D.J.; Nigg, B.M. Relationship between footwear comfort of shoe inserts and anthropometric and sensory factors. Med. Sci. Sports Exerc. 2001, 33, 1939-1945.

6. Mündermann, A.; Nigg, B.M.; Stefanyshyn, D.J.; Humble, R.N. Development of a reliable method to assess footwear comfort during running. Gait Posture 2002, 16, 38-45.

7. Luo, G.; Stergiou, P.; Worobets, J.; Nigg, B.; Stefanyshyn, D. Improved footwear comfort reduces oxygen consumption during running. Footwear Sci. 2009, 1, 25-29.

8. Lam, C.K.Y.; Mohr, M.; Nigg, S.; Nigg, B. Definition and quantification of 'ride' during running. Footwear Sci. 2018, 10, 77-82.

9. De Cock, A.; Vanrenterghem, J.; Willems, T.; Witvrouw, E.; De Clercq, D. The trajectory of the centre of pressure during barefoot running as a potential measure for foot function. Gait Posture 2008, 27, 669-675.

10. Kernozek, T.W.; Zimmer, K.A. Reliability and running speed effects of in-shoe loading measurements during slow treadmill running. Foot Ankle Int. 2000, 21, 749-752.

11. Fourchet, F.; Kelly, L.; Horobeanu, C.; Loepelt, H.; Taiar, R.; Millet, G.P. Comparison of plantar pressure distribution in adolescent runners at low vs. high running velocity. Gait Posture 2012, 35, 685-687.

(C) 2020 by the authors. Licensee MDPI, Basel, Switzerland. This article is an open access article distributed under the terms and conditions of the Creative Commons Attribution (CC BY) license (http://creativecommons.org/licenses/by/4.0/). 